\title{
Whisker Nuisance Test: A Valuable Tool to Assess Tactile Hypersensitivity in Mice Luigi Balasco ${ }^{1}$, Gabriele Chelini², $\$$, Yuri Bozzi ${ }^{1,3}$ and Giovanni Provenzano ${ }^{2, *}$
}

\author{
${ }^{1}$ Center for Mind/Brain Sciences (CIMeC), University of Trento, 38068 Rovereto, Italy; ${ }^{2}$ Department of \\ Cellular, Computational and Integrative Biology (CIBIO), University of Trento, 38123 Trento, Italy; ${ }^{3} \mathrm{CNR}$

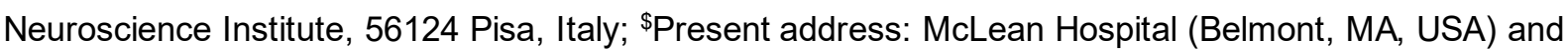 \\ Harvard Medical School (Boston, MA, USA) \\ *For correspondence: giovanni.provenzano@unitn.it
}

\begin{abstract}
[Abstract] Abnormal response to tactile stimulation, described as both hyper- and hypo-reactivity, is a common sensory impairment in multiple neuropsychiatric disorders. The neural bases of tactile sensitivity remain so far unknown. In the last years, animal studies have proven to be useful for shedding light on the cellular and molecular mechanism underlying sensory impairments. However, few behavioral tests have been developed in mice for assessing tactile perception abnormalities (e.g., the whisker nuisance [WN] test and the tactile prepulse inhibition assay). Here we provide a modified version of the WN test, which is based on the previously developed method by McNamara et al. (2010). The WN test permits to specifically detect tactile hypo/hyper-sensitivity relative to whisker stimulation in mice. The test starts with a habituation phase in which the mouse familiarizes itself with the experimental cage and the researcher/experimenter. After a sham session, the experimental session begins, consisting of bilateral whisker stimulation with a wooden stick. The advantages of using this protocol are many: it is relatively simple to set with no particular or expensive equipment needed, it is easily reproducible, it allows researchers to assess a variety of behavioral responses to a whisker-specific tactile perception in mice (i.e., fearful behavior, stance, hyperventilation, aggressive behavior and evasiveness) and provides important translational opportunities.
\end{abstract}

Keywords: Neurological Disorders, Sensory system, Tactile hypersensitivity, Mouse whiskers, Behavioral assessment

[Background] Over the last few decades, there has been a tremendous increase in research linking sensory processing dysfunction with different neuropsychiatric disorders (Hornix et al., 2019) such as autism spectrum disorder (ASD), fragile $X$ syndrome, post-traumatic stress disorder (PTSD), Rett Syndrome, schizophrenia, depression, anxiety and traumatic brain injury (TBI). It is interesting how typical cognitive difficulties of these neuropsychiatric disorders are more and more associated with alterations in the perception of the external world. One example of a specific sensory processing dysfunction that crosses the borders of several neuropsychiatric disorders is aberrant tactile sensitivity. Interestingly, it is estimated that about $90 \%$ of individuals diagnosed with ASD have atypical sensory experiences, described as both hyper- and hypo-reactivity, with abnormal responses to tactile stimulation (Marco et al., 2011).

Tactile sensory dysfunction is likely due to circuit dysfunction across the peripheral nervous system 
and the brain regions [e.g., primary somatosensory (S1) and thalamus] involved in processing and integration of tactile inputs (Hornix et al., 2019). Neural mechanisms underlying tactile sensitivity are not fully understood. However, recent studies using specific behavioral tasks and mouse model of neurological and neuropsychiatric disorders are considerably broadening our understanding of the neurobiological bases of tactile sensory impairments (McNamara et al., 2010, Orefice et al., 2016, He et al., 2017, Chelini et al., 2019). Moreover, the advent of next-generation approaches (i.e., optogenetics and chemo-genetics) in behavioral neuroscience have opened the door to test new hypotheses regarding the neural circuit involved in processing and integration of tactile inputs. To date only a limited number of behavioral tests have been developed for assessing tactile perception abnormalities in rodents [e.g., the whisker nuisance (WN) test and the tactile prepulse inhibition (tactile PPI) assay]. Therefore, there is a growing need for generating other behavioral tasks relevant to the tactile sensory dysfunctions.

One behavioral paradigm to evaluate tactile sensitivity in mice is the tactile PPI assay. This behavioral paradigm consists in delivering puffed air onto the back hairy skin of mice and is useful to specifically assess both hairy skin sensitivity and sensorimotor gating. A recent animal model study, by using the tactile PPI test, revealed how mice with mutations in Mecp2, Gabrb3, Shank3 or Fmr1 exhibit tactile hypersensitivity (Orefice et al., 2016).

Normal touch perception in the skin is mediated by the activation of cutaneous low-threshold mechanosensory neurons (LTMRs), which possess one peripheral axonal branch that innervates the skin and another branch that innervates the central nervous system (CNS). This task is valuable to study the circuit-level alterations related to the transmission of neuronal impulse between the LTMRs and the neurons of spinal cord that relay touch signaling to the sensory region of the brain.

However, sensory perception in mouse is also mediated by mystacial vibrissae (whiskers). Rodent whisker hair follicles are tactile organs functionally equivalent to human fingertips.

Rodent whiskers are exquisitely sensitive to touch and extract precise information about environment navigation, object recognition and social interactions. Afferent whisker information, via sensory nerve afferents, reaches layer IV of primary somatosensory cortex (SSp) for further processing (Petersen, 2007; Diamond et al., 2008).

Therefore, in our opinion, animal studies of tactile sensory processing must also consider whisker dependent tactile tasks, since whisker system is an important sensory organ essential to construction of the perceptual world.

Here, we provide a detailed and modified protocol of whisker nuisance (WN) test, to specifically assess tactile hypersensitivity relative to whisker stimulation in mouse. A previous $\mathrm{WN}$ test, considering seven different behavioral categories, was developed by McNamara and colleagues (McNamara et al., 2010) to assess sensory abnormalities in rats after diffuse brain injury. In rats, this test succeeded in demonstrating changes in sensory sensitivity after brain injury. Our modified version of WN test has revealed to be a valuable tool to evaluate whisker-dependent responses in mice in the absence of experimentally-induced lesions, as described in our recent work (Chelini et al., 2019).

We took advantage of Engrailed-2 knockout (En2-/-) mice, an informative model for understanding 
how neurodevelopmental defects can lead to cellular and circuit dysfunctions that directly or indirectly impact behaviors relevant to neuropsychiatric disorders.

Five different parameters are assessed during sensory stimulation in this test: fearful behavior, stance, breathing, response to stick and evasiveness. This test, together with previously developed sensory test, could represent a successful way to assess hyper-/hypo-sensitivity to sensory stimulation as well as to investigate the mechanisms underlying information processing during whisker-guided sensation in mouse, helping in shedding light to the sensory processing abnormalities found in human patients with neuropsychiatric and more in general with neurological disorders.

\section{Materials and Reagents}

1. Paper towels

Note: Paper towels and ethanol are used to clean the cage from excrements after habituation phase in the days before the proper testing.

2. Gloves

3. Adhesive paper tape

Note: The tape is useful to mark the position of the cage and the camera in the experimental room.

4. Laboratory mice: Each mouse strain which models a neurological disorder can be tested. Different life phases of mice can be considered to assess tactile hypersensitivity: young (1-3 months old), mature adult (3-6 months old), middle-aged (10-14 months) and old (18-24 months). Note: All mice tested should be of equal or similar age. Male mice should be tested separately in advance or in a different day since the smell of female mice could affect male mice behavior.

5. $70 \%$ ethanol $(\mathrm{v} / \mathrm{v})$

Note: Spray your hands with ethanol before and after handling of each mouse.

6. Odor-free disinfectant

Note: Spray all working surfaces with disinfectant after each testing session. Air dry for 2-3 min.

\section{Equipment}

1. Transparent standard mouse cage

Note: In our laboratory we use standard individual ventilated cage (Sealsafe Plus GM500, Tecniplast, Italy) with $501 \mathrm{~cm}^{2}$ floor area (W x D x H dimensions of $391 \times 199 \times 160 \mathrm{~mm}$ ). Any other transparent plastic cage with comparable measure can be used.

2. Video camera (LifeCam Studio 1080p HD Sensor/computer, Microsoft, CA), equivalent models from other companies can be used

Note: The quality of video recording should be higher enough (ideally 1,080p HD) to clearly assess single behaviors offline. The camera should be positioned $20-30 \mathrm{~cm}$ away from the cage in a position that allow to record the whole cage minimizing blind angles and reflexes (for 
reference see Figure 1). If recording light is present, cover it with paper tape. The raw data are stored in a computer for successive analysis.

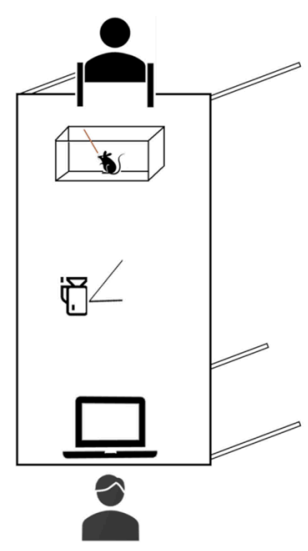

Figure 1. Representation of how the experimenters should position the camera and the experimental cage

3. Wooden stick (length: $20 \mathrm{~cm}$; diameter: $3 \mathrm{~mm}$ ) bought in any drug store Note: Blunt ends must be preferred to pointed ones in order to avoid possible physical harm.

4. Digital chronometer (Thermo Fisher Scientific-US, Fisherbrand ${ }^{\mathrm{TM}}$ Traceable $^{\mathrm{TM}}$ Jumbo-Digit Stopwatch), equivalent chronometer can be used

Note: Beeping noise from the chronometer should be avoided since could cause anxiety and fear in mice.

\section{Software}

1. Prism 8.0 (GraphPad, https://www.graphpad.com/) or similar software to perform statistical analysis

\section{Procedure}

See Figure 2 for the steps of the experimental procedure.

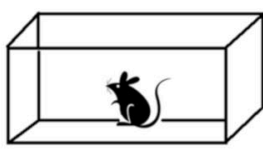

Sham

$35 \mathrm{~min}$

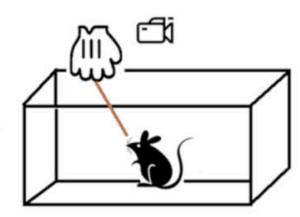

Habituation $5 \mathrm{~min}$

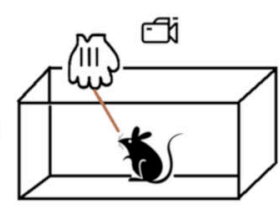

Test $3 \times 5 \mathrm{~min}$

Figure 2. Graphic representation of the behavioral testing steps. The behavioral testing consists of a habituation phase, in which the mice get used to the experimental cage, the novel environment and the experimenter. Habituation is then followed by sham-stimulation trial, where the 
wooden stick is introduced in the cage avoiding tactile contact with the animal. This will provide a baseline score of the animal anxious behavior (animals with high anxious response to the sham condition should be excluded from the WN test). Finally, the actual test phase will consist in bilateral stimulation of mice facial vibrissae.

Notes:

1. Each experimental mouse must be single-housed in a cage at least 1 week prior to beginning of behavioral testing. These cages are referred to as home cages.

2. Behavioral experiments must be performed in a dedicated room. Ideally, the behavioral room should have black curtains all around the experiment area in order to minimize the interference with environmental stimuli. The room must also be free of conspecific and heterospecific odors, since they can affect mouse behavior.

3. Noise should also be reduced to the minimum or to a comparable level of housing room. The experimenter must avoid making loud noises or sudden movements during both the habituation phase than the experimental phase.

4. The light should be reduced or at least indirect, however attention should be paid to the quality of video recording.

5. We recommend always running a pilot experiment to establish position of the camera and the cage and standardizing the experimental environment before starting the proper experiment.

6. Once the experimental conditions are standardized the experimenters can proceed with the behavioral testing.

A. Preparation of experimental cages (Day 0)

B. Prepare several clean cages equal to the number of mice you intend to test, these cages are referred to as experimental cages. Be sure to label all the cages properly since each mouse will have its own experimental cage.

C. Transfer a handful of bedding from the home cage to the experimental cage, close it and leave it for all the night. This step is necessary in order to facilitate the habituation to the novel environment. Be sure to clean your hands with alcohol each time you change cage.

Note: At any point during the experiment the investigator performing both stimulation and scoring of the test must be blinded from the experimental conditions. This procedure is easily applicable by preventing the experimenter from knowing the mice group of belonging.

D. Habituation phase (Days 1-3)

Choose a time for the habituation phase and the behavioral testing (i.e., morning), and then keep it for the duration of the whole experimental procedure. It is very important to be consistent in testing time parameters.

1. Remove the bedding from the experimental cage.

2. Transfer the mouse from its home cage to its experimental cage, without lid (Figure 3). 
Note: The cage should be placed in the same position every day; use adhesive tape to keep track of the correct position.

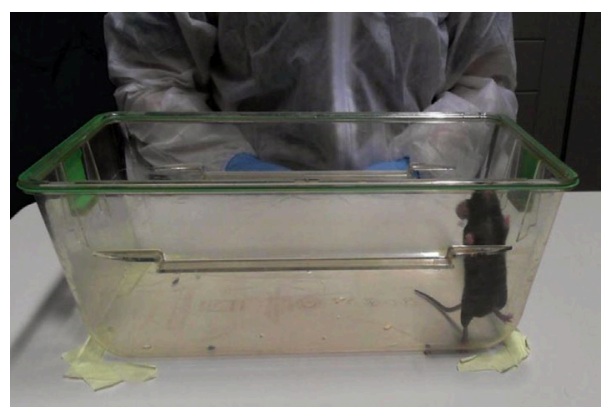

Figure 3. Mouse in the experimental cage during habituation phase

3. Leave the animal in the experimental cage for $30 \mathrm{~min}$. Be there, the animal should habituate also to your presence.

Note: The first day of habituation is the trickiest, the animals could be scared and jump out of the cage, pay attention and be patient.

4. At the end of the habituation phase transfer the mouse from the experimental cage to its home cage.

5. Remove all the urine and fecal boli from the experimental cage and clean with $70 \%$ ethanol.

6. Place a handful of bedding from the home cage to the experimental cage, close it and leave it for all the night.

7. On Day 2 repeat Steps B1 to B6.

8. On Day 3 repeat Steps B1 to B6.

Note: The habituation phase should be performed for at least 3 days, less days could have a negative impact on the behavioral assessment. A successful habituation is when the animals show no sign of fear, stress and/or anxiety (e.g., freezing, evasiveness, excessive grooming) due to the novel environment and to the presence of the experimenters.

E. Experimental phase (Day 4)

The experimental phase consists in the sham stimulation and in three trials of bilateral whisker stimulation. Both sham phase and stimulation phase last 5 min with 1 min of inter-trial interval.

1. Remove the bedding from the experimental cage.

2. Transfer the mouse from its home cage to its experimental cage, close the lid and position the cage in front of the camera.

3. Leave the animal in the experimental cage for $30 \mathrm{~min}$.

4. Open the lid of the cage and start recording

5. Start sham stimulation for 5 min (Video 1).

Note: In the sham stimulation, the animal is presented with the wooden stick. Pretend to stimulate the whisker of the mouse without touching them. 


\section{bïo-protocol

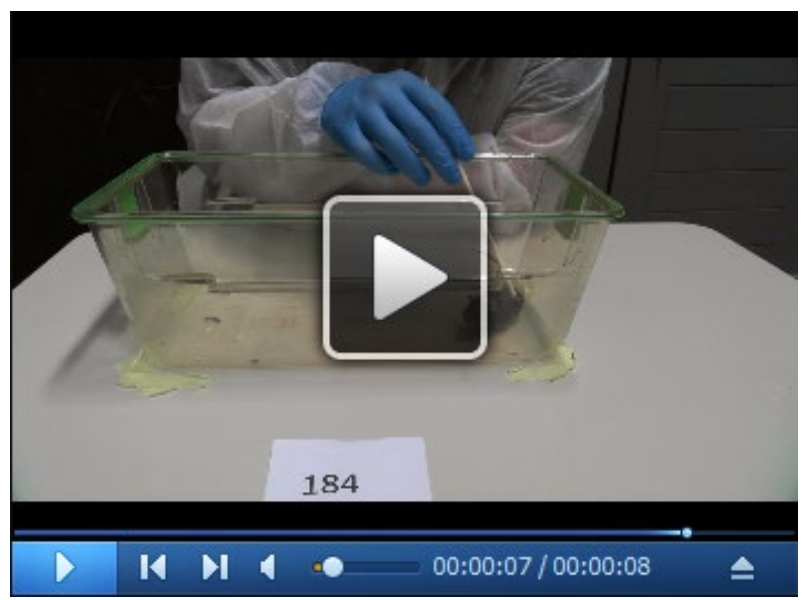

Video 1. Example of sham stimulation. (All animal procedures described in this protocol were approved by the University of Trento animal care committee and the Italian Ministry of Health (protocol 949/2015-PR).)

6. Start directly with trial 1 and continuously stimulate the whiskers for 5 min (Video 2).

Note. Use rhythmic movements to bend the middle of the whiskers and alternate the whisker stimulation (about 3 seconds in duration) for each side of the mouse's snout.

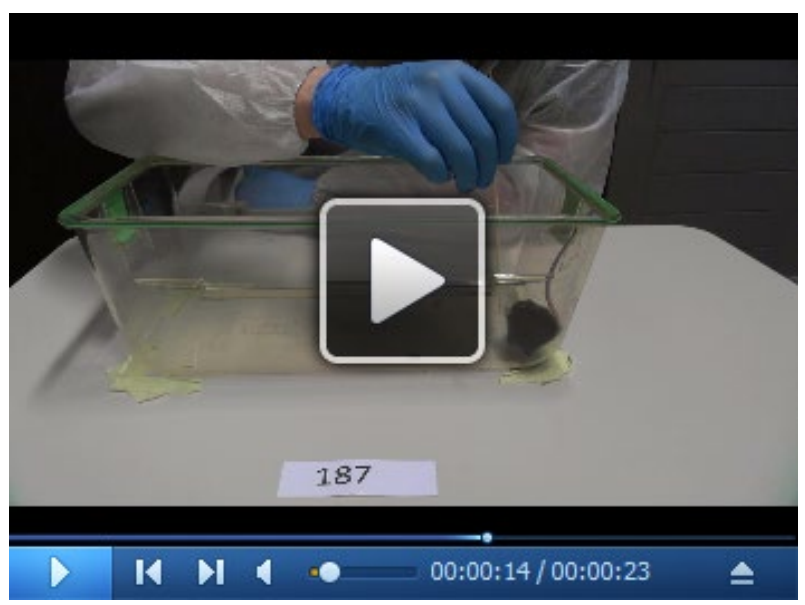

Video 2. Example of whisker stimulation. (All animal procedures described in this protocol were approved by the University of Trento animal care committee and the Italian Ministry of Health (protocol 949/2015-PR).)

7. Wait $1 \mathrm{~min}$.

8. Repeat Steps $\mathrm{C} 6$ and $\mathrm{C} 7$ other 2 times.

9. Stop the recording, save the video on a computer and go on with another mouse.

\section{Data analysis}

The behavioral assessment is based on manual video scoring of single behaviors. The behavioral 
assessment is made following Table 1. Five different categories are scored; the behavioral responses analyzed are fearful behavior (freezing, Video 3), stance (Video 4), breathing (hyperventilation, Video 5), aggressive response to stick presentation (Video 6) and evasiveness (Video 7 ) on a 0-2 points qualitative scale (0, absent; 1, scarcely present during the observation period; 2, present for most of the observation period).

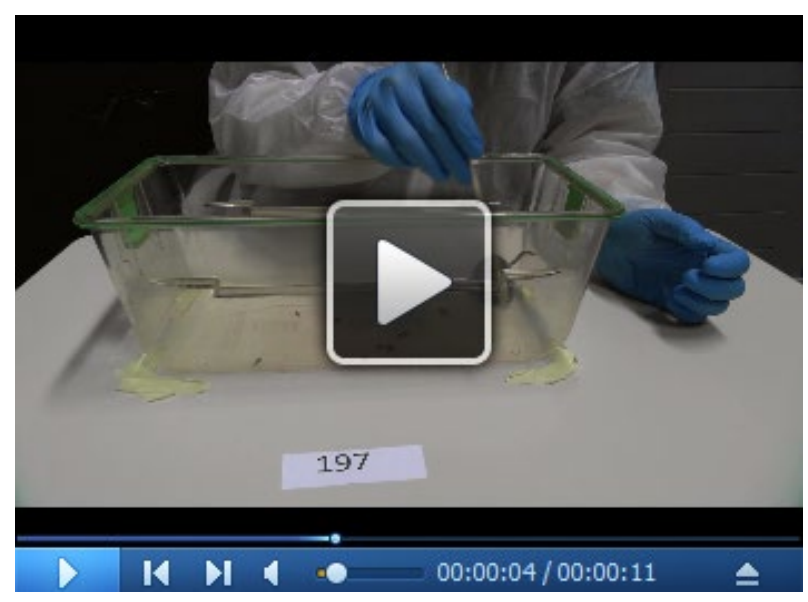

Video 3. Example of freezing. (All animal procedures described in this protocol were approved by the University of Trento animal care committee and the Italian Ministry of Health (protocol 949/2015PR).)

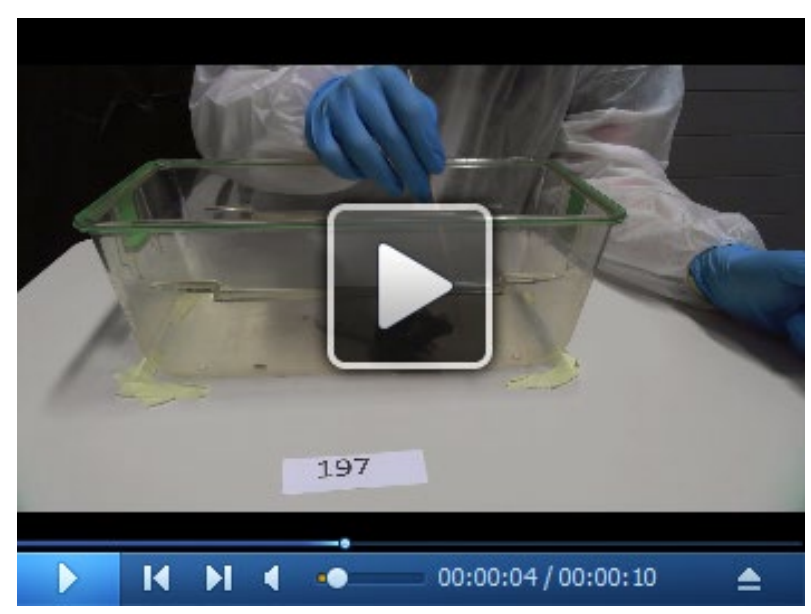

Video 4. Example of stance. (All animal procedures described in this protocol were approved by the University of Trento animal care committee and the Italian Ministry of Health (protocol 949/2015PR).) 


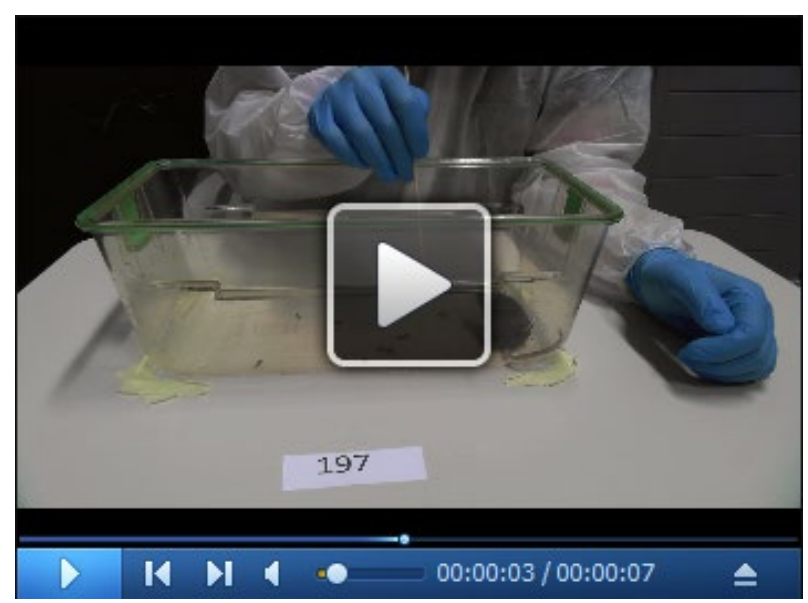

Video 5. Example of hyperventilation. (All animal procedures described in this protocol were approved by the University of Trento animal care committee and the Italian Ministry of Health (protocol 949/2015-PR).)

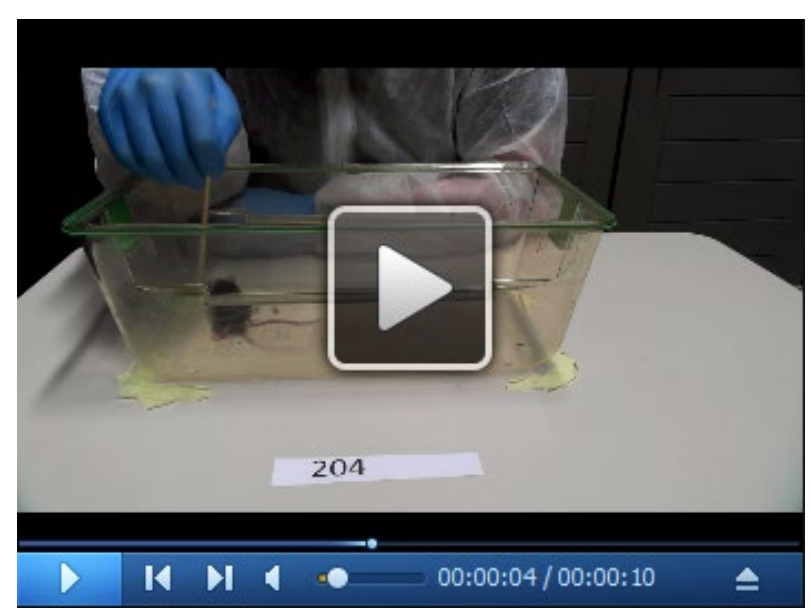

Video 6. Example of aggressive response to stick presentation. (All animal procedures described in this protocol were approved by the University of Trento animal care committee and the Italian Ministry of Health (protocol 949/2015-PR).)

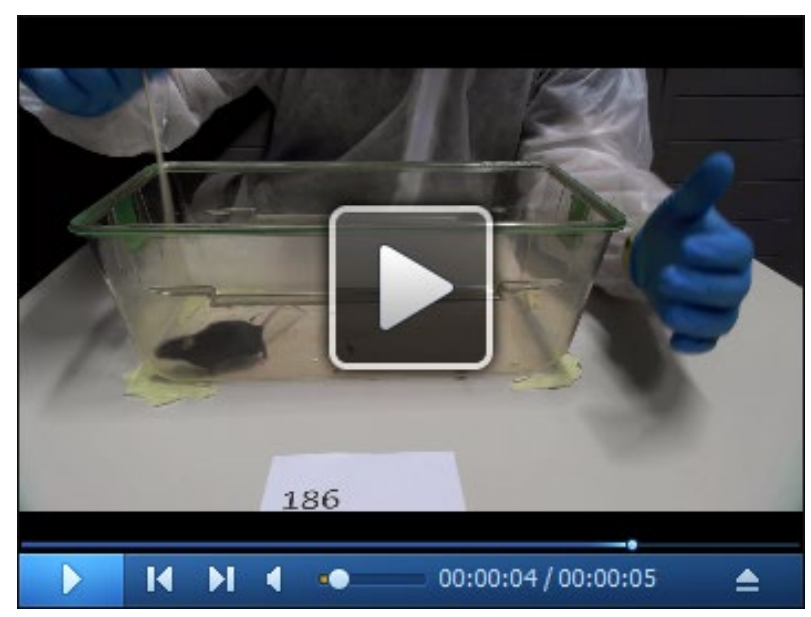

Video 7. Example of evasiveness. (All animal procedures described in this protocol were approved 
by the University of Trento animal care committee and the Italian Ministry of Health (protocol 949/2015-PR).)

Normal behavioral responses to stimulation are assigned a zero value, conversely meaningful abnormal behavioral responses (i.e., for most of the observation period) are assigned a value of 2 . The maximum score is 10 . This 10-point scale represents a modified version of the WN scoring scale by McNamara et al. (2010). High scores (8-10) indicate abnormal responses to the stimulation, in which the mouse freezes, becomes agitated or is aggressive. Low scores (0-3) indicate normal responses, in which the mouse is either curious or indifferent to the stimulation.

Table 1. The table shows the quantification of behavioral responses in the WN test. Five different parameters (fearful behavior, stance, breathing, response to stick, evasion) were monitored across test sessions (sham and trials 1-3). The total score indicates three major categories of behavioral responses ( 0 to 3 , curious/restful; 4 to 7 , annoyed/bothered; 8 to 10 , scared/worried).

\begin{tabular}{llll}
\hline & \multicolumn{3}{c}{ Score } \\
\cline { 2 - 4 } Behaviors & \multicolumn{1}{c}{$\mathbf{0}$} & $\mathbf{2}$ \\
\hline Fearful behavior & curious, insensible & ambivalent behavior & freezing \\
Stance & skyward head, relaxed & ambivalent behavior & guarded \\
Breathing & normal & ambivalent behavior & hyperventilated \\
Response to stick presentation & interested, ignore & ambivalent behavior & attacking, startle \\
Evasiveness & explorative behavior & ambivalent behavior & run away, avoiding \\
Total score & $\mathbf{0}$ to 3: curious, restful & $\mathbf{4}$ to 7: annoyed, & $\mathbf{8}$ to 10: scared, worried \\
\hline
\end{tabular}

Scoring should be performed at least by two trained independent operators to ensure a reliable scoring consistency. Statistical analysis can be conducted using GraphPad Prism 6.0 or any other statistical program and should be preferentially performed using analysis of variance (ANOVA), followed by appropriate post-hoc tests, with significance level set at $P<0.05$. We recommend using a minimum of 8-10 mice per genotype/group to reach statistical significance as well as to minimize the effects of inter-individual variation, although more may be necessary. 
We recently provided a sample analysis of behavioral response to whisker stimulation in mice lacking the ASD-related gene Engrailed-2 (En2 ${ }^{-1}$ ) mice (Chelini et al., 2019). En2 $2^{-1-}$ mice displayed a significantly higher score across the 3 trials, as compared with WT controls. When we analyzed the behavioral response of WT and En2-- mice for each of the five categories used to calculate the WN score, En2--mice displayed a significantly higher score in freezing and breathing, indicating a predominant fearful behavior of En2 mutants in response to repeated whisker stimulation. The reader is referred to Figure 5 of our original publication (Chelini et al., 2019) for details.

\section{Acknowledgments}

This behavioral protocol has been adapted from a previous study (McNamara et al., 2010), which succeeded in identifying sensory hypersensitivity to whisker stimulation in rats following diffuse brain injury. We thank the technical and administrative staff of $\mathrm{CIMeC}$ and $\mathrm{CIBIO}$ for excellent assistance. G.P. and Y.B. are currently supported by the University of Trento 2018-2020 Strategic Project "Trentino Autism Initiative-TRAIN".

\section{Competing interests}

The authors declare no conflict of interest.

\section{Ethics}

All animal procedures described in this protocol were approved by the University of Trento animal care committee and the Italian Ministry of Health (protocol 949/2015-PR).

\section{References}

1. Chelini, G., Zerbi, V., Cimino, L., Grigoli, A., Markicevic, M., Libera, F., Robbiati, S., Gadler, M., Bronzoni, S., Miorelli, S., Galbusera, A., Gozzi, A., Casarosa, S., Provenzano, G. and Bozzi, Y. (2019). Aberrant somatosensory processing and connectivity in mice lacking engrailed-2. $J$ Neurosci 39(8): 1525-1538.

2. Diamond, M. E., von Heimendahl, M., Knutsen, P. M., Kleinfeld, D. and Ahissar, E. (2008). 'Where' and 'what' in the whisker sensorimotor system. Nat Rev Neurosci 9(8): 601-612.

3. He, C. X., Cantu, D. A., Mantri, S. S., Zeiger, W. A., Goel, A. and Portera-Cailliau, C. (2017). Tactile defensiveness and impaired adaptation of neuronal activity in the fmr1 knock-out mouse model of autism. $J$ Neurosci 37(27): 6475-6487.

4. Hornix, B. E., Havekes, R. and Kas, M. J. H. (2019). Multisensory cortical processing and 
dysfunction across the neuropsychiatric spectrum. Neurosci Biobehav Rev 97: 138-151.

5. Marco, E. J., Hinkley, L. B., Hill, S. S. and Nagarajan, S. S. (2011). Sensory processing in autism: a review of neurophysiologic findings. Pediatr Res 69(5 Pt 2): 48R-54R.

6. McNamara, K. C., Lisembee, A. M. and Lifshitz, J. (2010). The whisker nuisance task identifies a late-onset, persistent sensory sensitivity in diffuse brain-injured rats. $J$ Neurotrauma 27(4): 695-706.

7. Orefice, L. L., Zimmerman, A. L., Chirila, A. M., Sleboda, S. J., Head, J. P. and Ginty, D. D. (2016). Peripheral mechanosensory neuron dysfunction underlies tactile and behavioral deficits in mouse models of ASDs. Cell 166(2): 299-313.

8. Petersen, C. C. (2007). The functional organization of the barrel cortex. Neuron 56(2): 339-355. 